\title{
Impact of PCB Manufacturing Process Variations on Trace Impedance
}

\author{
Abdelghani Renbi, Johan Carlson and Jerker Delsing \\ EISLAB, Department of Computer Science, Electrical and Space Engineering \\ Luleå University of Technology, SE-971 87 Luleå, Sweden \\ Abdelghani.Renbi@ltu.se,Johan.Carlson@ltu.se and Jerker.Delsing@ltu.se
}

\begin{abstract}
This paper demonstrates statistically the impact of $P C B$ manufacturing variations on the characteristic impedance. Moreover, it shows that the characteristics of the PCBs vary across different suppliers. These differences cannot be tolerated in some applications where the characteristic impedance is restricted to be within a specific range. We sampled $3 \times 20$ PCBs, each batch of twenty is ordered from a different manufacturer. The sampling consist of measuring the phase shift between the reflected and the incident signals when injecting a $180 \mathrm{MHz}$ sinewave into a PCB trace. The trace is selected to be the same for all samples. All the PCBs are ordered to be identical and designed for $50 \Omega$ devices. Our conclusion was drawn after running the T-tests to assess statistically the significance of the difference occurring between the PCBs. Based on the computed P-values all the three batches are different from each other in the mean of the measured phase shift with $95 \%$ confidence. The difference between the measured and the expected characteristic impedance is found as 3\%, $10 \%$ and $20 \%$ for these three manufacturers. We also witnessed board-to-board variations even within the same batch and from the same supplier due to the process instability by looking at the probability density of having the same phase shift that is equal to the mean. Some samples shown $2.6 \%$ to $3.5 \%$ difference above the mean.
\end{abstract}

Keywords: PCB; Trace; Characteristic Impedance; Manufacturing Process; Substrate; FR-4

\section{Introduction}

In high speed and RF applications, impedance mismatch has a significant impact on signal integrity and power loss while transferring a signal from source to destination due to the reflection. For sending signals over a line, the transmitter device must have an internal impedance which is equal to the characteristic impedance of the transmission line to avoid the reflections at the source. At the other end of the line, the receiver device must also have an internal impedance which is equal to the line characteristic impedance to avoid the reflections at the destination side. In the case of a mismatch, some of the signal is reflected back to its origin and reflections continue back and forth until the energy is fully absorbed, this can cause signal overshoot and undershoot which lead to digital state errors in high data rate applications.

After a careful PCB layout design, PCB manufacturing process can be responsible for low quality signal integrity measures. For demonstration, we compared three PCB manufacturing processes after measuring the phase shift between the incident and the reflected signals when injecting a $180 \mathrm{MHz}$ sinewave into a PCB trace. Each process is presented by 20 PCB samples of the same design. We also evaluated the process stability for each manufacturer [1].

\section{Related Work}

The impacts of the fabrication process have been studied in [2], the focus was about the impact of copper surface roughness and the moisture absorbed by the pre-preg on the conductive and the dielectric loss respectively. Copper surface roughness is achieved by the adhesion promotion treatment. As the copper roughness worsens the conductive loss, a balance between the mechanical reliability and the conductive loss is needed, the mechanical reliability refers to the mechanical joint between the copper surface and the pre-preg which is ensured by the copper surface roughness. The other outcome of the research is that the mois- 
ture absorbed by the pre-preg before the lamination process degrades eternally the dielectric loss. Precautions are to be taken in account to achieve good storing conditions which minimize pre-preg moisture absorption.

In [3], where the author studied the the impact of PCB weave on the skew. Looking at the substrate from the weave level, the traces lie on non-homogenous dielectric, therefore some traces might be on the top of a substrate where the the epoxy resin is dominant and others might be on the top of the substrate where the fiber glass is dominant, this causes the wave to propagate with different speeds within the same board and therefore a skew between an equal length traces. The weave can lead to $5 \%$ to more than $10 \%$ variation in propagation delay within identical physical length. The percentage of glass to epoxy and weave style have a significant impact on dialectic constant and therefore on the propagation delay.

PCB fabricators are facing big challenges to meet good quality for high frequency requirements, short pulses technique has been published in [4] for PCB manufacturers to assess the produced PCB trace in term of frequency dependent propagation constant, this can increase the confidence of the manufacturers about their quality by running few tests.

\section{PCB manufacturing variations and char- acteristic impedance}

\subsection{Characteristic impedance}

Generally, the characteristic impedance is a complex number with a resistive and reactive component. It is a function of the frequency of the applied signal, and is independant to length. Based on the telegrapher's equations the characteristic impedance is:

$$
Z_{0}=\sqrt{\frac{R+j L \omega}{G+j C \omega}}
$$

Where $R$ is the resistance per unit length, $L$ is the inductance per unit length, $G$ is the conductance of the dielectric per unit length, $C$ is the capacitance per unit length, $j$ is the imaginary unit, and $\omega$ is the angular frequency.

Talking about the same length of a PCB trace. At the same frequency, any change in the input impedance signifies a change in the capacitance for that specific length which formed by the the trace and the ground plane with the insulator. Any change in per unit length capacitance signifies a change in the characteristic impedance. By measuring the changes in the input impedance of a trace we will also be measuring a change in the characteristic impedance.

\subsection{Possible manufacturing variations}

There exist several variations that can occur during volume production of PCBs, many of these variations will affect the characteristic impedance of the trace and may fall outside the accepted range. Figure 1 shows a typical PCB trace which is called a microstrip line.

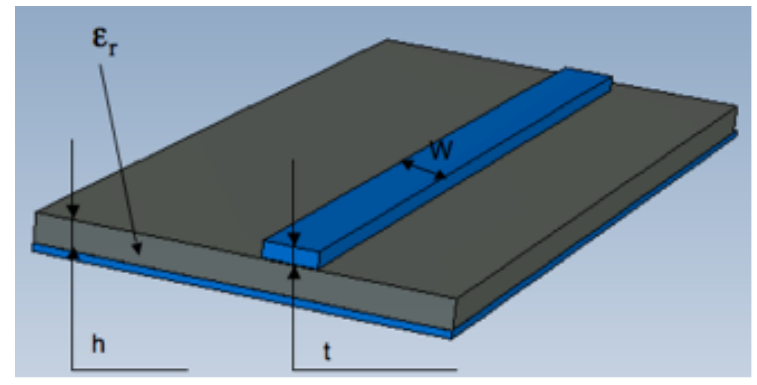

Fig. 1: PCB trace.

The ratio of the conductor width $\frac{W}{h}$ to the distance from the power plane $h$ plays an important role in controlling the impedance, $\varepsilon_{r}$ which is the dielectric constant of the material that separates the trace from the power plane is also an important factor. These two work together to form the characteristic impedance described in the equation 1.

Variations in etching affect the width of the trace which results in changes in the area, typically the tolerance on trace width is $\pm 10 \%$. The processes used for creating the core FR-4 and for pressing the pre-preg layers of FR-4 are also inexact.

The thickness of a trace is determined by the employed copper foil and is rarely specified with a tolerance, mostly is considered constant. The dielectric constant may be in the range of 4.2 to 4.4 when is intended to be 4.3.

In practice, a PCB trace is not rectangular as intended to be. Process differences between suppliers, between different batches of PCBs or even within a single batch from one supplier, can cause significant variations in the shape and smoothness of a trace perimeter.

All these variations will enforce the characteristic impedance to be within a range while is expected to be $50 \Omega[1]$.

\section{Methodology}

As mentioned in section 3.1, Any change in per unit length capacitance signifies a change in the characteristic impedance. By measuring the changes in the input impedance of a trace, see figure 2, we will also be measuring a change in the characteristic impedance.

To measure the change in the input impedance we measured the phase shift of the reflected sig- 
nal from the input impedance formed by the PCB trace after injecting a $180 \mathrm{MHz}$ sinewave signal.

The measurements have been performed in a stable environment and any significant deviations in the measured values have been repeated several times to ensure the validity of data. The frequency has been chosen to be specifically $180 \mathrm{MHz}$ for adjusting the measuring device to its highest sensitivity, this frequency depends on the coaxial line length which has been employed for signal transmission.

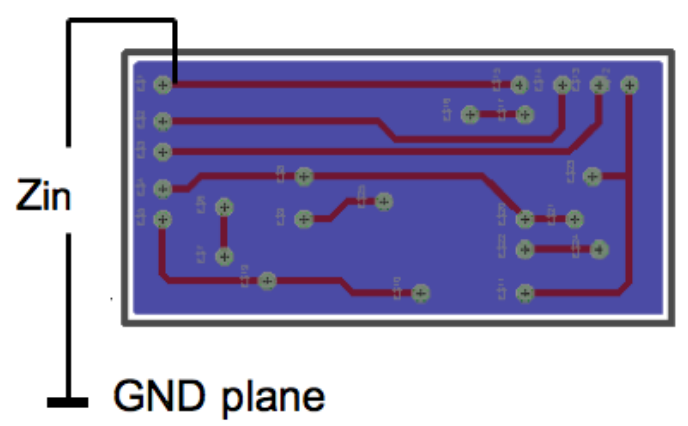

Fig. 2: Employed trace for process evaluation. It is designed for $50 \Omega$ devices.

Following table represents the collected data from the three manufacturers samples.

TABLE I: Phase shift data.

\begin{tabular}{|c|c|c|c|}
\hline $\begin{array}{c}\text { PCB } \\
\text { samples }\end{array}$ & M1 & M2 & M3 \\
\hline \hline $\mathbf{1}$ & 2.33 & 2.27 & 2.30 \\
\hline $\mathbf{2}$ & 2.34 & 2.27 & 2.32 \\
\hline $\mathbf{3}$ & 2.32 & 2.27 & 2.32 \\
\hline $\mathbf{4}$ & 2.33 & 2.27 & 2.30 \\
\hline $\mathbf{5}$ & 2.31 & 2.26 & 2.31 \\
\hline $\mathbf{6}$ & 2.32 & 2.27 & 2.31 \\
\hline $\mathbf{7}$ & 2.32 & 2.25 & 2.33 \\
\hline $\mathbf{8}$ & 2.34 & 2.26 & 2.29 \\
\hline $\mathbf{9}$ & 2.42 & 2.27 & 2.32 \\
\hline $\mathbf{1 0}$ & 2.34 & 2.27 & 2.32 \\
\hline $\mathbf{1 1}$ & 2.33 & 2.27 & 2.32 \\
\hline $\mathbf{1 2}$ & 2.32 & 2.27 & 2.38 \\
\hline $\mathbf{1 3}$ & 2.34 & 2.28 & 2.32 \\
\hline $\mathbf{1 4}$ & 2.34 & 2.27 & 2.31 \\
\hline $\mathbf{1 5}$ & 2.34 & 2.26 & 2.32 \\
\hline $\mathbf{1 6}$ & 2.34 & 2.26 & 2.38 \\
\hline $\mathbf{1 7}$ & 2.34 & 2.26 & 2.30 \\
\hline $\mathbf{1 8}$ & 2.34 & 2.26 & 2.32 \\
\hline $\mathbf{1 9}$ & 2.35 & 2.27 & 2.31 \\
\hline $\mathbf{2 0}$ & 2.35 & 2.26 & 2.32 \\
\hline
\end{tabular}

\section{Analysis}

\subsection{T-test and P-value}

In statistical analysis, the most commonly used procedure to determine whether or not two populations have different mean values on some measure is the T-test procedure. Let A and B be the two populations in question and $\mathrm{M}$ is the measure. We might do a research to clarify the hypothesis that $\mathrm{A}$ and $\mathrm{B}$ are different regarding the measure M. We run an experiment or a questionnaire to measure some samples of $\mathrm{M}$ in both of populations. We assume that the null hypothesis is true, which means that there is no difference between $\mathrm{A}$ and $\mathrm{B}$ on the measure $\mathrm{M}$. What will happen if we observe a difference in the mean values of $\mathrm{M}$ in both populations? How will we decide that the populations A and B are really different?

The T-test allows us to answer the above questions by computing the $\mathrm{P}$-value that indicates the strength of the evidence against the null hypothesis. If the $\mathrm{P}$-value is less than 0.05, the null hypothesis is to be rejected and the difference is statistically significant. If the $\mathrm{P}$-value is greater than 0.05 , the difference between the two populations is not statistically significant.

\subsection{Significance of mean differences}

Based on the results which are shown in table I, we can see clearly that there is a difference between the PCBs. By looking at table II we may capture an image about the significance of the difference between the three batches. To draw a conclusion whether or not the batches of the PCBs are physically different from each other, we ran the T-tests to assess statistically the significance of the difference occurring between the batches. We posed an hypothesis $H_{0}$, which claims that each batch has an equal mean $\mu$ as the other one. According to the computed P-Values, which are shown in table III, all these three batches are different and the hypothesis is rejected with less than $5 \%$ chance.

TABLE II: Phase shift mean and StDev values.

\begin{tabular}{|c|c|c|}
\hline Manufacturer & Phase shift mean $\mu$ & StDev \\
\hline \hline M1 & 2.338 & 0.02215 \\
\hline M2 & 2.266 & 0.00681 \\
\hline M3 & 2.320 & 0.02271 \\
\hline
\end{tabular}

\subsection{Characteristic impedance verification}

In this section, we evaluate the expected quality of these three batches of PCBs. The specified requirement of the $\mathrm{PCB}$ trace is to be $50 \Omega$ as the characteristic impedance. For this we performed the scattering parameters extraction using a vector network analyzer and computed the charac- 
TABLE III: P-values with $95 \%$ CI for mean difference.

\begin{tabular}{|c|c|c|}
\hline $\begin{array}{c}\text { Mean } \\
\text { difference }\end{array}$ & P-Value & $\begin{array}{c}\text { 95 \% CI for } \\
\text { mean difference }\end{array}$ \\
\hline \hline$\mu_{1}-\mu_{2}$ & 0.000 & $0.06163,0.08237$ \\
\hline$\mu_{1}-\mu_{3}$ & 0.024 & $0.00269,0.03331$ \\
\hline$\mu_{2}-\mu_{3}$ & 0.000 & $-0.06530,-0.04270$ \\
\hline
\end{tabular}

teristic impedance within a range of frequency of $100 \mathrm{MHz}$ up to $1.5 \mathrm{GHz}$. To compute the characteristic impedance with sufficient accuracy, the de-embedding procedure has been taken into the consideration to remove the effect of the connector which we used for the measurement:

$$
\left[\begin{array}{ll}
A & B \\
C & D
\end{array}\right]_{D U T}=\left[\begin{array}{ll}
A & B \\
C & D
\end{array}\right]_{\text {Port }}^{-1} \times\left[\begin{array}{cc}
A & B \\
C & D
\end{array}\right]_{\text {Device }}
$$

The device matrix represents the matrix of the cascaded connector with the trace as shown in the upper part of figure 3 . The port matrix represents the matrix of the connector. The DUT matrix represents the matrix of the trace as they are separated in the lower part of figure 3 . The complete followed procedure is well described in [5].

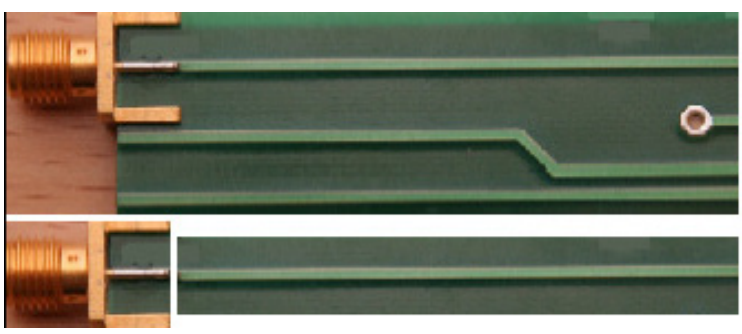

Fig. 3: Device, Port and DUT.

After computing the ABCD matrix of the trace which is refereed as device under test (DUT), directly we computed the characteristic impedance which can be written as:

$$
Z_{c}=\sqrt{\frac{A}{C} \frac{B}{D}}=\sqrt{\frac{B}{C}}
$$

when the system trace is symmetric as it is in our case.

Geometrically, the trace has been designed to be $49.8 \Omega$ with 4.2 as the FR- 4 epoxy dialectic constant. According to the results shown in the figure 4, The process of M1 led to the closet characteristic impedance as intended to be, while the process of M3 resulted on a moderate quality, on the other hand a significant difference is shown in the characteristic impedance of trace from M2, this latest might lead to unexpected behavior in high frequencies applications.

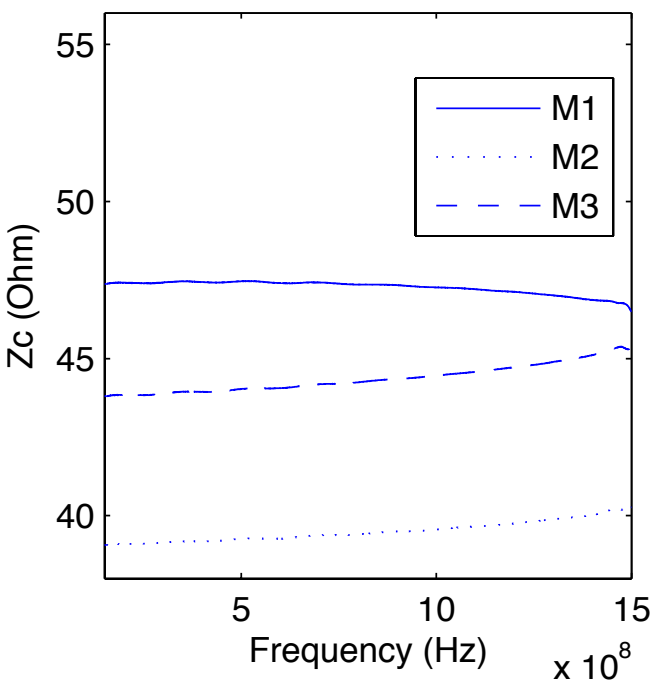

Fig. 4: Characteristic impedance from the three processes.

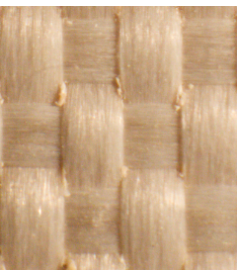

(a) M1

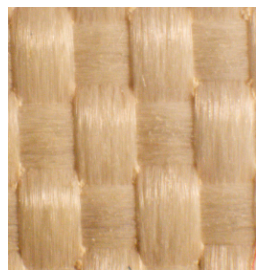

(b) M2

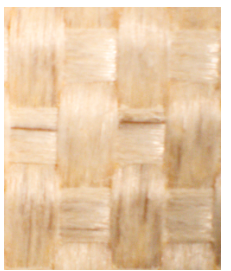

(c) M3
Fig. 5: Glass cloth after the dissolving process

\subsection{Glass cloth weave}

After the dissolving process of the epoxy , the weave of the glass cloth illustrated in figure 5 used by M1, M2 and M3 seems to be similar therefore the weave cannot be the main contributor to difference obtained in the phase shift results especially when the trace is $1 \mathrm{~mm}$ wider. We could expect some differences due to the cloth weave in the PCB used by M3 if the trace width is much smaller that the bundle width where it might lie on different dielectric constant of the substrate composite. This comparison put the thickness of the substrate and the tolerances of the dielectric constant of both epoxy and glass in a good position of the source of the difference in the resulted phase shift values.

\subsection{Stability analysis}

Figure 6 shows the the probability density of measuring the same phase shift between the reflected and the incident signal been applied to the trace within the batch of PCBs from M1, M2 and M3. The phase shift is represented here by a voltage quantity according to the the measuring device.

The process of M2 is characterized by the high- 


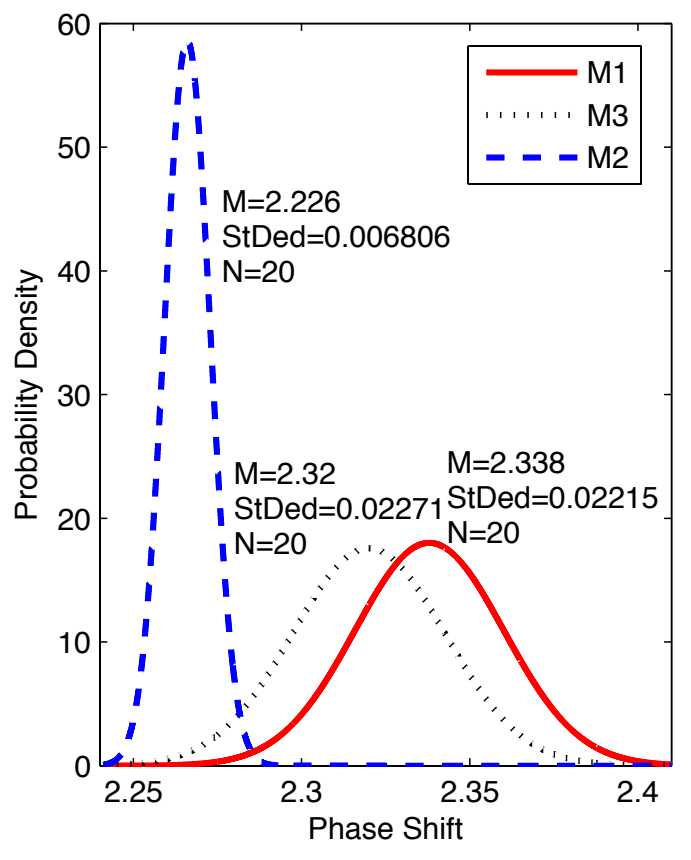

Fig. 6: Probability density of the phase shift.

est stability as all the samples shown almost similar phase shift with a very small standard deviation of $\sigma=0.006806$, thus the process is a good example for nearly identical PCBs production. On the other hand, both processes of the other two manufacturers are less stable. M1 process led to a phase shift standard deviation of $\sigma=0.02215$, one sample of its population shown an increase by almost $3.51 \%$ above the mean. This sample has been repeated several times to ensure the confidence of our conclusions. This confirms what we discussed in section 3.2 about the probable difference within a batch of PCBs which are produced by the same process. The biggest factor candidate which might be responsible of this difference could be thickness of the sample due to some mechanical variations while pressing the pre-preg layers. M3 process is the least stable with a standard deviation of $\sigma=0.02271$ in the phase shift. There are two samples which are notable to be above the mean $\mu=2.32$ of the phase shift by $2.6 \%$. This also part of the possible variations within the same produced batch.

Figure 7 shows the the probability density of measuring the same phase shift between the reflected and the incident signal been applied to the trace within the all the batches of PCBs from all manufacturers. The phase shift is represented by a voltage quantity according to the the measuring device.

According to the graph, the standard deviation of the phase shift $\sigma=0.03593$ got higher as compared to the one we obtained within the same pro-

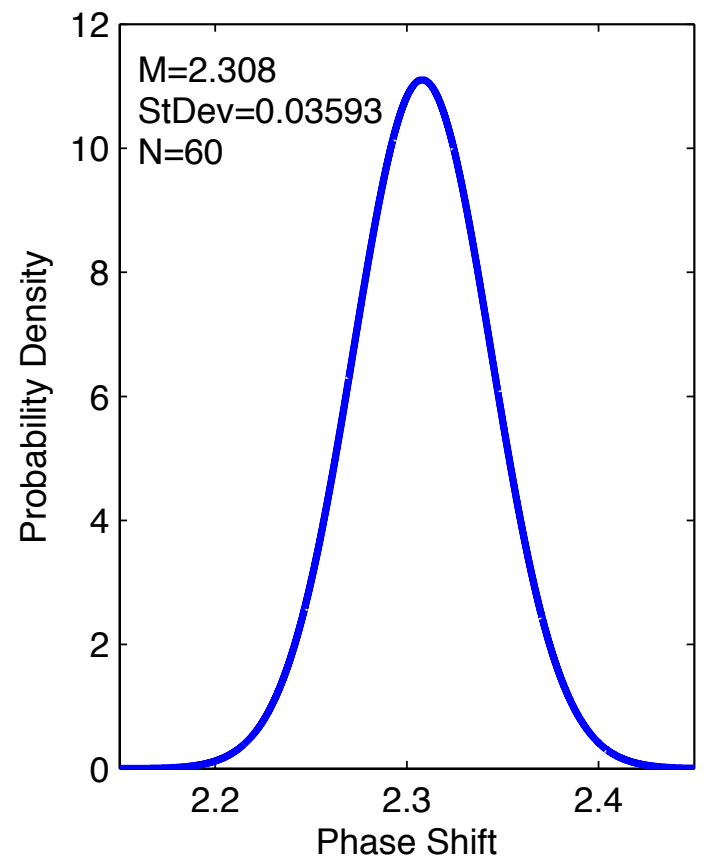

Fig. 7: Probability density of the phase shift mean in design from all three manufacturers.

cess, this explains that the process are different from each others and one should consider whether or not the difference can be tolerated while changing the PCB supplier for a specific design [6].

\section{Conclusion}

Statistical analysis shows that PCB characteristic impedance might be out of the tolerated range of a specific RF design due to the instability of the design process. For extremely critical RF applications, extra caution need to be considered when ordering from different PCB suppliers. Statistical analysis can be very useful to evaluate PCB vendors quality before making big orders.

\section{References}

[1] C. F. Coombs, Printed Circuits Handbook, 5th ed. McGraw-Hill Professional Publishing, 2007, ch. 10.

[2] O. H. S. C. Jason Lin, Albert Huang, "A case study of the impact of fabrication processes on pcb broadband electrical characteristics." International Symposium on Microelectronics, 2009.

[3] S. McMorrow, "The impact of pcb lmainate weave on the electrical performance of differential at multigigabit data rates," 2005.

[4] R. K. John DiTucci, "Production measurement of frequency dependent attenuation and phase constant of pcb traces." IMAPS, 2009.

[5] S. H. Hall, Advanced Signal Integrity for High-Speed Digital Designs, 1st ed. Wiley-IEEE Press, 2009, ch. 9.

[6] D. C. Montgomery, Design and Analysis of Experiments, 7 th ed. Wiley, ch. 2. 